\title{
Glycosphingolipids and Sphingolipids Closely Associated with or Causing Apoptosis
}

\author{
Sen-itiroh Hakomori and Yasuyuki Igarashi \\ The Biomenbrane Institute, 201 Elliott Ave W, Seattle, WA 98119; and Depts. of Pathobiology and Microbiology, \\ University of Washington, Seattle, WA98195
}

\begin{abstract}
Key words: Apoptosis (programmed cell death), Glycosphingolipid (Le ${ }^{y}$ GSL, Le ${ }^{x}$ GSL and Gb3), Sphingolipid (ceramide and sphingosines), Canbohydrates, Cell death signaling pathway
\end{abstract}

\section{Background}

Glycosphingolipids (GSLs) are ubiquitous membrane components found in all animal cells and most plant cells, including yeasts. GSLs play crucial functional roles in cell-cell recognition, cell adhesion, and modulation of transmembrane signaling [13]. Patterns of GSL expression change dramatically during the processes of ontogenesis, oncogenesis, and differentiation of stem cells into phenotypically terminal stage cells. Our knowledge of GSL distribution and expression was greatly enriched by introduction of the monoclonal antibody approach, and many developmentally-regulated, tumor-associated GSL antigens have been identified on a chemical basis [12, 14].

Apoptosis (programmed cell death) is a fundamental physiological process in all multicellular organisms. It helps control tissue size, shape, and balance of cell number during morphogenesis, and plays an important role in oncogenic progression $[2,31]$ and in other pathological processes such as reperfusion injury [11] and glomerulonephritis [3]. In view of the well-established changes of GSL expression which occur in conjunction with ontogenesis and oncogenesis, we and others have investigated the relationship between apoptosis and GSL expression patterns. Strong associations have been observed between apoptosis and expression of $\mathrm{Le}^{\mathrm{y}}, \mathrm{Le}^{\mathrm{x}}$, and $\mathrm{Gb} 3 \mathrm{Cer}$ in human normal and tumor tissues. Apoptosis can be triggered by exogenous addition of various GSLs (or their degradation products such as ceramides and sphingosines), suggesting that GSL expression changes are a cause, rather than an effect, of apoptosis.

Here, we will briefly review our current knowledge of this exciting research area, and point our some remaining problems to be solved.

Presented in part at the workshop 3 on Le ${ }^{y}$ Antigen, A Marker for Apoptosis: Diagnosis and Prognosis of Cancer, July 14, 1994 as part of the program at the 4th Joint Meeting of the Japan Society of Histochemistry and Cytochemistry and the histochemical Society, held in Maui, Hawaii, July 13 to 16, 1994.

\section{Expression of $\mathbf{L e}^{\mathrm{y}}$ Antigen in Relation to Apoptosis in Epithelial Cells and Tumors Derived Therefrom}

Hiraishi et al. [16] reported a close correlation between Le $\mathrm{L}^{\mathrm{y}}$ expression (defined by mAb BM1) and apoptosis in various tumor tissues and normal epithelial tissues. Apoptosis was assessed not only by typical morphological features (e.g. shrinkage of cytoplasm, condensation of nucleus) but also by nick-end labeling for detection of DNA fragmentation [10]. Some important findings from this study were: (i) apoptotic cells were detected frequently in $\mathrm{Le}^{\mathrm{y}}$-positive cell populations but not $\mathrm{Le}^{\mathrm{y}}$-negative populations; (ii) cells that were Ley-positive were usually PCNA (proliferating cell nuclear antigen)-negative, nickend labeling positive, and showed apoptotic morphology; (iii) apoptotic morphology was not strongly correlated with positive staining for Fas antigen (CD95/Apo-1). These trends were observed in normal and tumor tissues from esophagus, colon, and stomach, and in normal thymus.

$\mathrm{Le}^{\mathrm{y}}$ antigen is known to be present in both GSLs and glycoproteins, particularly those having mucin-type domains $[1,26]$. Structures of Le ${ }^{y}$ GSL have been identified as \#s 1, 2, and 3 below.

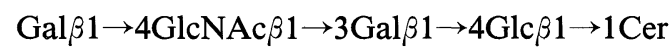

2

$$
3
$$

Fuc $\alpha 1 \quad$ Fuc $\alpha 1$

2
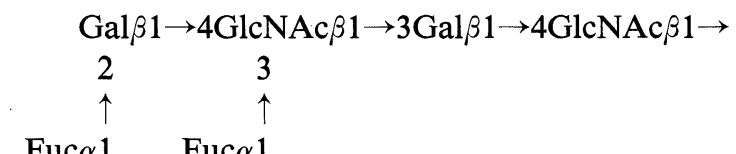

$$
3 \mathrm{Gal} \beta 1 \rightarrow 4 \mathrm{Glc} \beta 1 \rightarrow 1 \mathrm{Cer}
$$

3

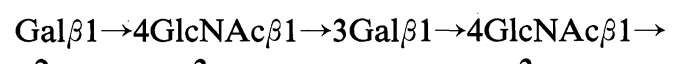

$\begin{array}{rrr}2 & 3 & 3 \\ \uparrow & \uparrow & \uparrow \\ \text { Fuc } \alpha 1 & \text { Fuc } \alpha 1 & \text { Fuc } \alpha 1 \\ 3 \mathrm{Gal} \beta 1 & \rightarrow 4 \mathrm{Glc} \beta 1 \rightarrow 1 \mathrm{Cer} & \end{array}$


We suspect that only Ley GSL (not Le ${ }^{y}$ glycotprotein) antigen is involved in apoptosis. Which of the three $\mathrm{Le}^{\mathrm{y}}$ GSL structures shown above preferentially causes apoptosis is unknown. During differentiation, the carbohydrate structure $\mathrm{Le}^{\mathrm{y}}$ may define the stage of cells committed to apoptosis (see "Discussion" and Fig. 1 legend).

\section{Expression of Globotriaosylceramide (Gb3 /CD77) in Relation to Apoptosis in B Cell Population and Burkitt Lymphoma}

Globotriaosylceramide (Gb3) is expressed highly in Burkitt lymphoma, and was long known as "Burkitt lymphoma antigen" (BLA) before being chemically identified as below:

$$
\mathrm{Gal} \alpha 1 \rightarrow 4 \mathrm{Gal} \beta 1 \rightarrow 4 \mathrm{Glc} \beta 1 \rightarrow 1 \mathrm{Cer}
$$

This structure is also expressed highly in certain B cell populations (where it has been recently termed "CD77"), particularly in germinal centers of tonsils and other lymphoid tissues which exhibit active apoptosis. It has been detected at lower levels in a variety of other cells and tissues. Gb3 is also abundant in individuals of histoblood group $\mathrm{P}^{\mathrm{K}}$ (a rare phenotype seen in about $0.01 \%$ of most populations).

A correlation between apoptosis and Gb3/CD77 expression in B cell populations was first demonstrated by a morphological study employing electron micrography [23]. The fact that Gb3/CD77 functions as a receptor for Shiga toxin [17] and verotoxin (VT) [21] suggests that toxin-dependent killing of $\mathrm{Gb3} / \mathrm{CD} 77(+)$ cells may depend on apoptosis. A recent study showed that the Bsubunit of VT (VT-B), which causes binding of VT to Gb3 $/ \mathrm{CD} 77$, also induces apoptosis in $\mathrm{Gb3} / \mathrm{CD} 77(+)$ cells. $\mathrm{Gb} 3$ glycolipid, when bound to VT-B, is internalized and creates some signal to induce DNA fragmentation [24]. Furthermore, when $\mathrm{Gb3} / \mathrm{CD} 77(+)$ cells were treated with VT-B, a Gb3/VT-B complex was accumulated at the nuclear membrane. This complex may induce DNA fragmentation via signaling across the nuclear membrane (see "Discussion").

\section{Induction of Apoptosis by Ceramide and Sphingolipid Derivatives}

In human monocytoblastic leukemia U937 cells, hydrolysis of sphingomyelin are resulting generation of ceramide (Cer) have been implicated in signal transduction leading to induction of apoptosis by tumor necrosis factor $\alpha(\mathrm{TNF} \alpha)$ [18] and, very recently, by Fas antibody [5]. Cer itself has also been claimed to induce apoptosis [27]. Cer has no effect on protein kinase C. On the other hand, some cells have been reported to contain Cer-activated protein kinases or phosphatases which induce apoptotis signaling $[6,22,25]$.

In view of these earlier findings, we compared the effects of Cer (C-2, C-6, C-8 cell-permeable ceramide), sphingosine (Sph), N,N-dimethyl-Sph (DMS), and Sph-1- phosphate (Sph-1-P) on apoptosis in a number of cell lines (leukemia, solid cancer-derived, normal 3T3, etc.). All these compounds are produced during the "sphingomyelin/glycosphingolipid cycle" (note: we believe that this phrase is more accurate than "sphingomyelin cycle"). We observed that: (1) In the presence of regular serum, in most cancer cell lines, DMS was the strongest inducer of apoptosis, followed by Sph. In normal cells such as BALB/c 3T3 (A31) or HUVEC, late G1 arrest of cell cycle (but not apoptosis) was produced by DMS and sph. Cellpermeable Cer had a negligible effect on apoptosis [30]. (2) In the absence of serum, the effect of Cer in human leukemia (U937, HL60) cells increased, as reported previously by others [27], but not to the degree seen for DMS of Sph [30]. (3) Induction of apoptosis in human neutrophils by $\mathrm{TNF} \alpha$ was clearly correlated with increased level of Sph, and exogenously-added Sph corresponding to this level (10-20 $\mu \mathrm{M})$ induced apoptosis [28]. Similar results were observed in phorbol ester-induced apoptosis of HL60 cells [29].

\section{Discussion}

Apoptosis is an important phenotypic change observed in multicellular organisms as a counterpart of cell proliferation, differentiation, maturation, and oncogenesis [31]. Dramatic changes in membrane glycosylation are correlated with differentiation and oncogenesis. We hypothesize that specific glycosylation changes are similarly associated with apoptosis. We previously observed that apoptosis is associated with increased $\mathrm{Le}^{\mathrm{y}}$ antigen expression, decreased expression of PCNA, and increased nickend labeling (reflecting DNA degradation) [16].

Despite extensive studies of various normal and tumor tissues, the mechanistic connection between Le ${ }^{y}$ expression and apoptosis remains unknown. Le $\mathrm{Le}^{\mathrm{y}}$ can be carried by both glycoproteins and GSLs. In view of a general relationship of apoptosis with sphingolipid changes [23-25, 27-30], we assume that expression of Le ${ }^{\mathrm{y}}$-bearing GSLs is involved in apoptosis. There is some evidence for involvement of $\mathrm{Le}^{\mathrm{x}}$ expression in apoptosis in tumor tissues [Tachikawa T, kannagi R, pers. comm.], but the number of cases studied is small compared to our studies of $\mathrm{Le}^{\mathrm{y}}$ expression. One credible hypothesis is that GSLs bearing $\mathrm{Le}^{\mathrm{y}}$ or $\mathrm{Le}^{\mathrm{x}}$ are internalized preferentially over other GSLs because of their stronger tendency to aggregate and form clusters. Internalization of these GSLs leads to a "chain reaction" of degradation to Cer or Sph at lysosomes, switching on of signal transduction, and a new phosphorylation pathway (Fig. 1). The presence of endoglycosylceramidase in animal cells and tissues was recently reported $[4,20]$.

Apoptosis in epithelial cells and tumors derived therefrom is correlated with expression of $\mathrm{Le}^{\mathrm{y}}$ and $\mathrm{Le}^{\mathrm{x}}$. No such correlation is seen in lymphoid cells or B cell lymphoma. Rather, apoptosis in these lymphoid cells is correlated with expression of $\mathrm{Gb3} / \mathrm{CD} 77$, the same antigen 


\section{Lex Epithelial cell \\ Ley \\ Epithelial cell}
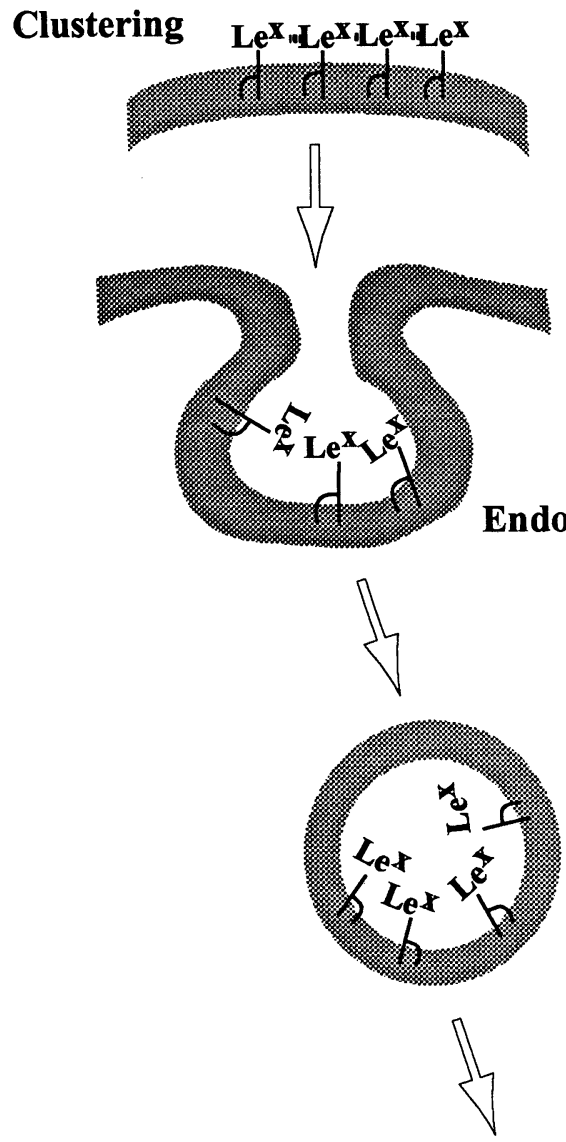

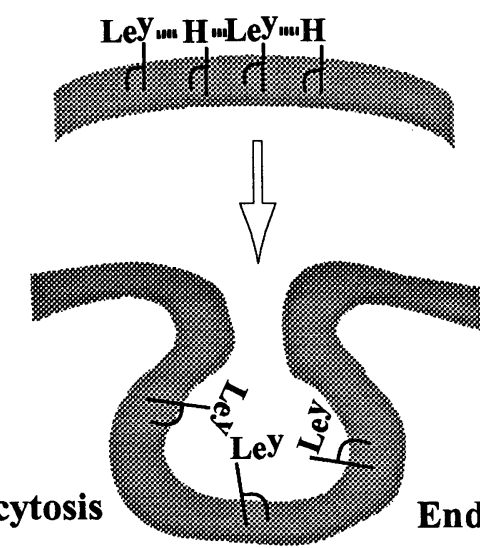

Endocytosis
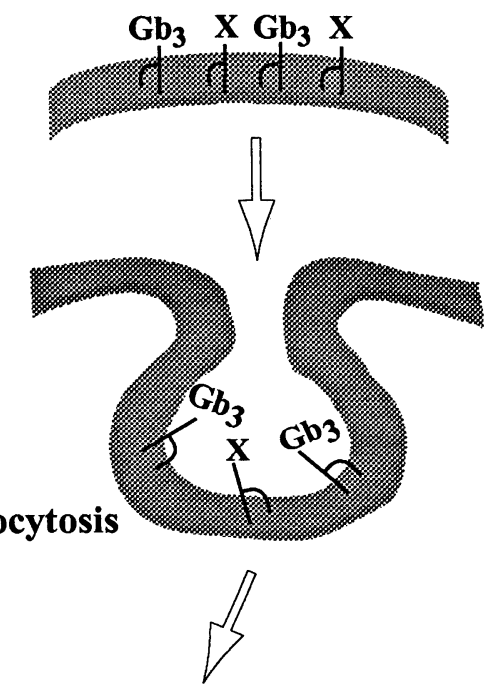

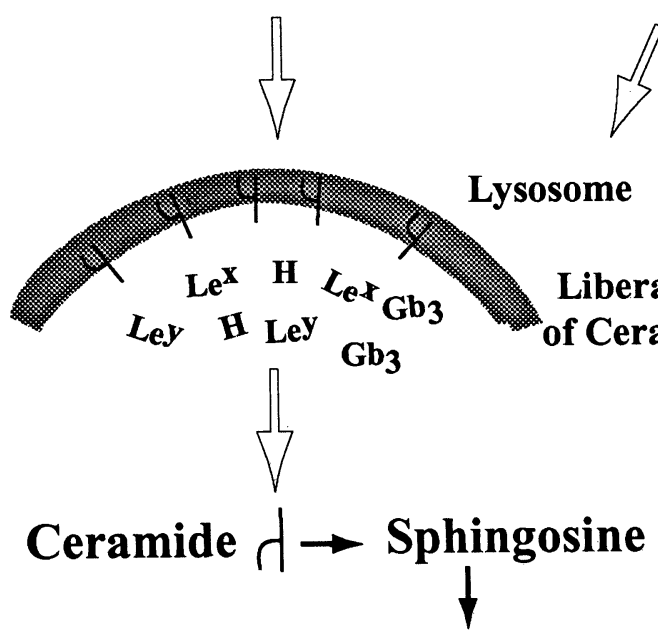

N,N-Dimethylsphingosine

Fig. 1. Possible mechanism for induction of apoptosis by cell type-specific GSLs.

GSLs having limited types of specific structure, such as Ley, Gb3, and (to some extent) Le ${ }^{\mathrm{x}}$, have been found to be associated with apoptosis. There is some evidence that internalization of specific GSLs helps trigger apoptosis. A conceptual scheme based on our present knowledge is shown here. Le ${ }^{y}$ and H GSLs are associated with and interact with each other [9]. Clusters of H-Ley complex are formed and internalized by endocytosis. Le $\mathrm{e}^{\mathrm{x}}$ clusters are formed by $\mathrm{Le}^{\mathrm{x}}-\mathrm{Le}^{\mathrm{x}}$ interaction [7] and similarly internalized. It is possible that Gb3 interacts with SLe $\mathrm{S}^{\mathrm{x}}$ (Nudelman ED, Hakomori S, unpubl. data) to form clusters which are then internalized. However, a complementary binding counterpart of $\mathrm{Gb} 3$ to form clusters has not been conclusively identified. It is known that VT-B promotes Gb3 internalization (see text). GSL clusters internalized as described above are degraded to Cer and oligosaccharides by endoglycosylceramidase at lysosomes. The presence of endoglycosylceramidase in animal cells has been demonstrated $[4,20]$, and this enzyme is probably associated with lysosomes in analogy to various glycolytic and proteolytic enzymes. Released Cer is subsequently converted to Sph and DMS, which strongly induce apoptosis through a conjoint pathway with TNF $\alpha$ (see Fig. 2). 
responsible for rare blood group $\mathrm{P}^{\mathrm{K}}$ (see above). Thus, tissue- or cell type-specific occurrence of apoptosis is probably triggered by high accumulation of specific types of GSL.

Accelerated internalization of VT-B, which binds only to $\mathrm{Gb} 3$ and has no toxic effect by itself, has been clearly shown to induce apoptosis [24]. Since Gb3/VT-B complex is detected at the nuclear membrane of apoptotic cells [Lingwood CA, pers. comm., and presented at the June 1994 meeting of the Federation of European Biochemical Societies], we can hypothesize that this complex is subsequently degraded and induces endonuclease activation and DNA fragmentation.

Studies of apoptosis-related GSL antigens are so far limited to $\mathrm{Le}^{\mathrm{y}}, \mathrm{Le}^{\mathrm{x}}$, and $\mathrm{Gb} 3$; i.e. no extensive studies have been performed on other GSL species. Association of specific GSL species with apoptosis is probably highly cell type- and tissue type-specific. Carbohydrates which define cell type may also define the differentiation stage where apoptosis occurs. However, apoptosis itself may be triggered not by carbohydrates but rather by Cer or sphingolipids, as suggested by a number of recent studies $[15,19,27-30]$.

Our current concept (based on results from this laboratory and others) of the relationship between sphingolipids and various cellular functions, particularly apoptosis, is summarized in Fig. 2. The most well-studied and widely-occurring cell surface receptors which induce apoptosis are the receptors for $\mathrm{TNF} \alpha$ [25] and Fas [5]. These two receptors show 26\% sequence homology with each other in a stretch of 65 amino acids. Cer and Nmethyl-Sph induce apoptosis, and TNF $\alpha$ induces elevation of Sph and Cer levels, as described above. Sphingolipids are therefore assumed to be the messengers triggered by

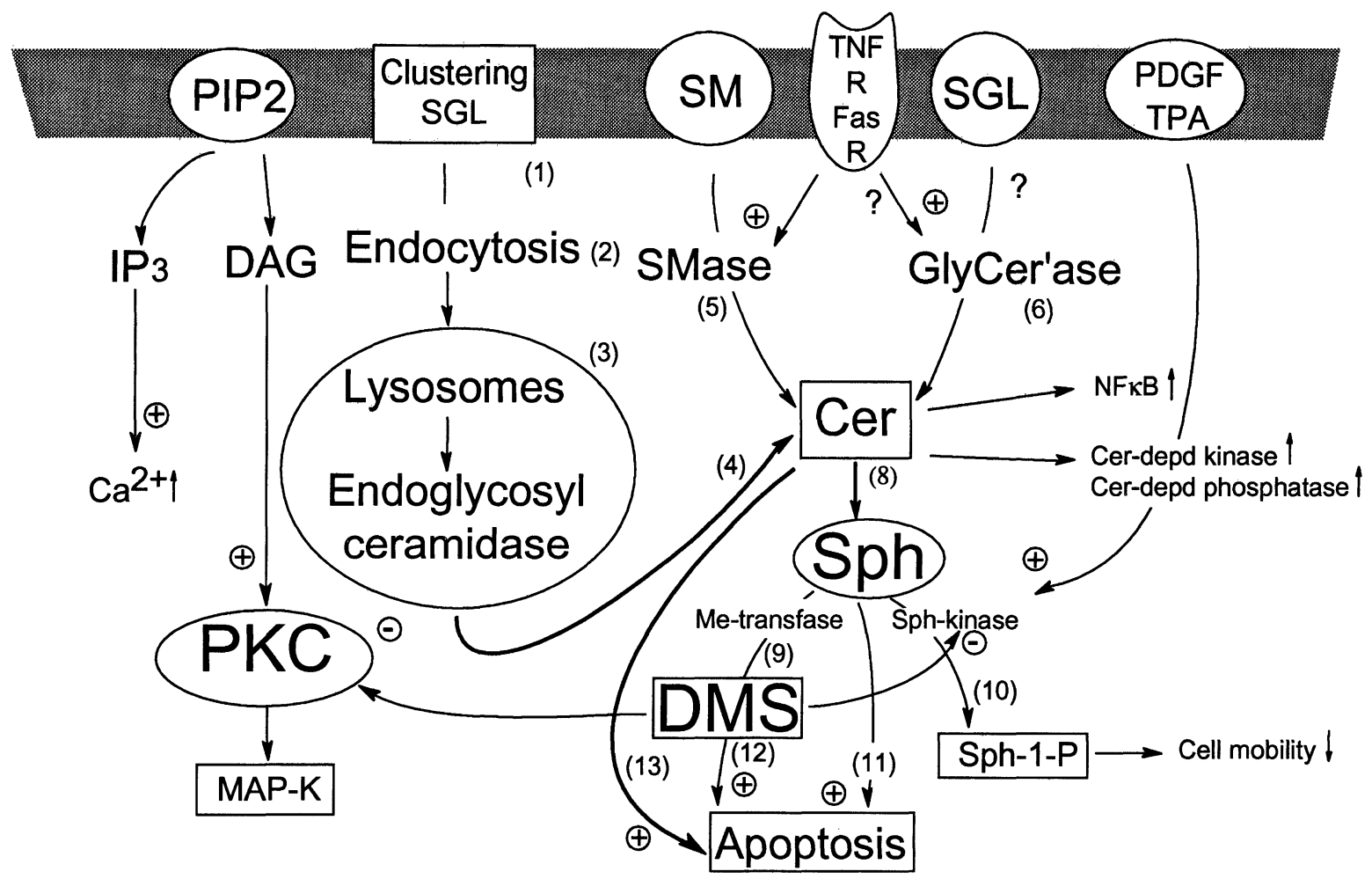

Fig. 2. Cell signaling pathway, affected by GSLs and sphingolipids, which induce apoptosis, cell growth, and cell motility.

As a consequence of clustering of GSLs [1], followed by endocytosis [2] and degradation of GSLs at lysosomes [3], Cer is liberated [4] (this same process is shown in Fig. 1). Cer can also be derived from sphingomyelin (SM) by activation of sphingomyelinase [5] or possibly from GSL by activation of endoglycosylceramidase [6], although this latter route remains to be clarified. Processes 5 and (possibly) 6 are activated by an unknown mechanism through signal transduction via Fas or TNF receptor [7]. Ceramide is further degraded by ceramidase to sphingosine (Sph) [8] which is in turn methylated to form N,N-dimethylsphingosine (DMS) [9], or phosphorylated by Sph kinase to Sph-1phosphate (Sph-1-P) [10]. Apoptosis is strongly induced by Sph [11] or DMS [12], but less strongly by Cer [13]. Thus, liberation of Cer [3, 4, $5,6]$, consequent liberation of Sph, and conversion of Sph to DMS [8, 9] are of central importance for inducing apoptosis [11, 12, 13], although the mechanism of apoptosis induction through Sph and DMS is not known. Cell proliferation is controlled by PKC, possibly through MAP kinase (MAP-k), PKC activity is down-regulated by DMS but up-regulated by diacylglycerol (DAG). Cell motility is controlled by Sph-1-P. In summary, PKC and MAP-k affect mainly cell proliferation, while Sph-1-P affects mainly cell motility. Since actively-proliferating (PCNA-positive) cells are never undergoing apoptosis, the apoptosis-inducing effect of Sph and DMS may be ascribable to their inhibitory effect on PKC through myc down-regulation. This idea is compatible with the observation that apoptosis is always associated with differentiation and growth inhibition (see text). 
TNF $\alpha$ and (probably) Fas, although the mechanism for activation of NF- $\kappa \mathrm{B}$ or other transcription factors, and its relationship to endonuclease activation, remains unknown.

Apoptosis is an essential physiological process which helps regulate cell numbers in normal as well as tumor tissues. If rate of apoptosis is enhanced in tumor cells, total tumor mass will decrease. This is a potentially useful approach to anti-tumor therapy. We previously demonstrated that DMS, a strong inducer of apoptosis, inhibits tumor growth in nude mice [8].

At each step of normal organogenesis and morphogenesis, apoptosis plays a key role in defining morphological adaptation for proper functioning of tissues and organs. It is well-documented that each step of development and differentiation involves major changes in cell surface glycosylation. Many of the carbohydrates involved are bound to sphingolipids. Furthermore, carbohydrate residues of sphingolipids play important roles in cell recognition, both adhesive and repellant. We hypothesize that, in analogy to $\mathrm{Le}^{\mathrm{y}}, \mathrm{Le}^{\mathrm{x}}$, and Gb3, sphingolipids in general help control two important and distinct processes: cell recognition (when located at the cell surface) and apoptosis (following internalization). Normal ontogenesis represents a delicate balance between these two processes, both of which are regulated by changing expression of specific glycosphingolipids at each stage.

\section{Acknowledgment}

We thank Dr. Stephen Anderson for scientific editing and preparation of the manuscript.

\section{References}

1. Abe, K., McKibin, J. M. and Hakomori, S.: The monoclonal antibody directed to difucosylated type 2 chain (Fuc $\alpha 1 \rightarrow$ 2 Gal $\beta 1 \rightarrow 4[$ Fuc $\alpha 1 \rightarrow 3]$ GlcNAc; Y determinant). J. Biol. Chem. 258; 11793-11797, 1983.

2. Arends, M. J. and Wyllie, A. H.: Apoptosis: Mechanism and roles in pathology. Int. Rev. Exp. Pathol. 32; 223-254, 1991.

3. Baker, A. J., Mooney, A., Hughes, J., Lombardi, D., Johnson, R. J. and Savill, J.: Mesangial cell apoptosis: The major mechanism for resolution of glomerular hypercellularity in experimental mesangial proliferative nephritis. J. Clin. Invest. 94; 2105-2116, 1994

4. Basu, S., Ghosh, S., Basu, M., Hawes, J. W., Das, K. K., Zhang, B., Li, Z., Weng, S. and Westervelt, C.: Carbohydrate and hydrophobic-carbohydrate recognition sites (CARS and HY-CARS) in solubilized glycosyltransferases. Ind. J. Biochem. Biophys. 27; 386-395, 1990.

5. Cifone, M. G., De Maria, R., Roncaioli, P., Rippo, M. R., Azuma, M., Lanier, L. L., Sántoni, A. and Testi, R.: Apoptosis signaling through CD95 (Fas/Apo-1) activates an acidic sphingomyelinase. J. Exp. Med. 177; 1547-1552, 1994.

6. Dobrowsky, R.T. and Hannun, Y.A.: Ceramide-activated protein phosphatase: Partial purification and relationship to protein phosphatase 2A. Adv. Lipid Res. 25; 91-104, 1993.

7. Eggens, I., Fenderson, B. A., Toyokuni, T., Dean, B., Stroud, M. R. and Hakomori, S.: Specific interaction between $\mathrm{Le}^{\mathrm{x}}$ and
Le $^{\mathrm{x}}$ determinants: A possible basis for cell recognition in preimplantation embryos and in embryonal carcinoma cells. $J$. Biol. Chem. 264; 9476-9484, 1989.

8. Endo, K., Igarashi, Y., Nisar, M., Zhou, Q. and Hakomori, S.: Cell membrane signaling as target in cancer therapy: Inhibitory effect of $N, N$-dimethyl and $N, N, N$-trimethyl sphingosine derivatives on in vitro and in vivo growth of human tumor cells in nude mice. Cencer Res. 51; 1613-1618, 1991.

9. Fenderson, B. A., Kojima, N., Stroud, M. R., Zhu, Z. and Hakomori, S.: Specific interaction between $\mathrm{Le}^{\mathrm{y}}$ and $\mathrm{H}$ as a possible basis for trophectoderm-endometrium recognition during implantation [Abstract 8.5]. Glycoconj. J. 8; 179, 1991.

10. Gavrieli, Y., Sherman, Y. and Ben-Sasson, S. A.: Identification of programmed cell death in situ via specific labeling of nuclear DNA fragmentation. J, Cell Biol. 119; 493-501, 1992.

11. Gottlieb, R. A., Burleson, K. O., Kloner, R. A., Babior, B. M. and Engler, R. L.: Reperfusion injury induces apoptosis in rabbit cardiomyocytes. J. Clin. Invest. 94; 1621-1628, 1994.

12. Hakomori, S.: Aberrant glycosylation in tumors and tumorassociated carbohydrate antigens. Adv. Cancer Res. 52; 257331, 1989.

13. Hakomori, S.: Bifunctional role of glycosphingolipids: Modulators for transmembrane signaling and mediators for cellular interactions. J. Biol. Chem. 265; 18713-18716, 1990.

14. Hakomori, S. and Kannagi, R.: Glycosphingolipids as tumorassociated and differentiation markers. J. Natl. Cancer Inst. 71; 231-251, 1983.

15. Hannun, Y. A. and Linardic, C. M.: Sphingolipid breakdown products: Anti-proliferative and tumor-suppressor lipids. Biochim. Biophys. Acta. 1154; 223-236, 1993.

16. Hiraishi, K., Suzuki, K., Hakomori, S. and Adachi, M.: Le $\mathrm{Le}^{\mathrm{y}}$ antigen expression is correlated with apoptosis (programmed cell death). Glycobiology 3; 381-390, 1993.

17. Jacewicz, M., Clausen, H., Nudelman, E. D., Donohue-Rolfe, A. and Keusch, G. T.: Pathogenesis of Shigella diarrhea: XI. Isolation of a Shigella toxin-binding glycolipid from rabbit jejunum and HeLa cells and its identification as globotriaosylceramide. J. Exp. Med. 163; 1391-1404, 1986.

18. Jarvis, W. D., Kolesnick, R. N., Fornari, F. A., Traylor, R. S., Gewirtz, D. A. and Grant, S.: Induction of apoptotic DNA damage and cell death by activation of the sphingomyelin pathway. Proc. Natl. Acad. Sci. USA 91; 73-77, 1994.

19. Kolesnick, R. N. and Golde, D. W.: The sphingomyelin pathway in tumor necrosis factor and interleukin-1 signaling. Cell 77; 325-328, 1994.

20. Li, Z., Hawes, J. W., Tang, H., Basu, M. and Basu, S.: Heat stability study of rabbit mammary ceramide glycanase (CGase) activity by a novel assay [Abstract]. FASEB J. 6; A492, 1992.

21. Lingwood, C. A., Law, H., Richardson, S., Petric, M., Brunton, J. L., De Grandis, S. and Karmali, M.: Glycolipid binding of purified and recombinant Escherichia coli produced verotoxin in vitro. J. Biol. Chem. 262; 8834-8839, 1987.

22. Lozano, J., Berra, E., Municio, M. M., Diaz-Meco, M. T., Dominguez, I., Sanz, L. and Moscat, J.: Protein kinase C $\zeta$ isoform is critical for $\kappa \mathrm{B}$-dependent promoter activation by sphingomyelinase. J. Biol. Chem. 269; 19200-19202, 1994.

23. Mangeney, M., Richard, Y., Couland, D., Tursz, T. and Wiels, J.: CD77: An antigen of germinal center B cells entering apoptosis. Eur. J. Immunol. 21; 1131-1140, 1991.

24. Mangeney, M., Lingwood, C. A., Taga, S., Caillou, B., Tursz, T. and Wiels, J.: Apoptosis induced in Burkitt's lymphoma cells via $\mathrm{Gb} 3 / \mathrm{CD} 77$, a glycolipid antigen. Cancer Res. 53; 5314-5319, 1993.

25. Mathias, S., Dressler, K. A. and Kolesnick, R. N.: Characterization of a ceramide-activated protein kinase: Stimulation by tumor necrosis factor $\alpha$. Proc. Natl. Acad. Sci. USA 88; 
10009-10013, 1991.

26. Nudelman, E. D., Levery, S. B., Kaizu, T. and Hakomori, S.: Novel fucolipids of human adenocarcinoma: Characterization of the major $\mathrm{Le}^{\mathrm{y}}$ antigen of human adenocarcinoma as triducosylnonaosyl $\mathrm{Le}^{\mathrm{y}}$ glycolipid ( $\mathrm{III}^{3} \mathrm{FucV}^{3} \mathrm{FucVI}^{2} \mathrm{FucnLc}_{6}$ ). J. Biol. Chem. 261; 11247-11253, 1986.

27. Obeid, L. M., Linardic, C. M., Karolak, L. A. and Hannun, Y. A.: Programmed cell death induced by ceramide. Science 259; 1769-1771, 1993.

28. Ohta, H., Yatomi, Y., Sweeney, E. A., Hakomori, S. and Igarashi, Y.: A possible role of sphingosine in induction of apoptosis by tumor necrosis factor- $\alpha$ in human neutrophils. FEBS Lett. 355; 267-270, 1994.

29. Ohta, H., Sweeney, E. A., Masamune, A., Hakomori, S. and
Igarashi, Y.: Induction of apoptosis by sphingosine in human leukemic HL-60 cells: A possible endogenous modulator of apoptotic DNA fragmentation occurring during phorbol ester-induced differentiation. Cancer Res. 55; 691-697, 1995.

30. Sweeney, E. A., Ohta, H., Hakomori, S. and Igarashi, Y.: Sphingosine and its methylated derivatives cause apoptosis in a variety of human and mouse cancer cell lines: A comparison of their effects and those of cell permeable ceramide analogues [Abstract, Mar 1995 AACR meeting, Toronto, Canada]. AACR proceeding $36 ; 15$, A85.

31. Wyllie, A. H.: Cell death: A new classification separating apoptosis from necrosis. In "Cell death in biology and pathology," ed. by I. D. Bowen and R. A. Lockshin, Chapman \& Hall, London, 1981, pp. 9-34. 